\title{
An optimization analysis of the rapidity and maneuverability of an inland SWATH-USV
}

\author{
Zhanyuan Cheng, Songlin Yang, Chengqi Cai, Wenpeng Cai, Xiaoyu Huang
}

\begin{abstract}
In this paper, we designed a kind of SWATH-USV through corresponding ship hull parameters selected as design variables to identify the SWATH performance as Optimal objective function and constraints for rapidity and maneuverability of SWATH. Via a multidisciplinary optimization design and analysis software, we analyzed and compared the single optimization of rapidity and the comprehensive optimization of rapidity and maneuverability. By comparing the fitness function values of the chaos algorithm and the hybrid algorithms of discrete genetic iterations, it was found that the genetic algorithm of $\mathbf{5 0 0 0}$ generations combined with comprehensive optimization of rapidity and maneuverability has the highest value, and the optimal optimization results of ship form parameters were generated.
\end{abstract}

Index Terms- SWATH-USV; Multi-disciplinary comprehensive optimization; Chaos algorithm; genetic algorithm

\section{INTRODUCTION}

In the 21 st century, mankind has entered a period of large-scale development and utilization of the ocean. Oceans play a more important role in the pattern of national economic development and opening up, and play a more prominent role in safeguarding national sovereignty, security and development interests. China is a developing country with both land and sea. Building a strong maritime country is an important part of building a strong socialist modernization country in an all-round way.

As a high performance ship, SWATCH has the advantages of wide deck area, good sea-keeping and transverse stability, and low resistance in high-speed navigation. It has become a hot ship form in recent years. Therefore, the optimization technology of ship form parameters of SWATCH has become increasingly hot. SHIPFLOW and NASTRAN are mainly used to demonstrate and study ship types at home and abroad. Wei Keke proposed a new type of small water surface wave-piercing hybrid catamaran $^{[1]}$, and analyzed it through resistance calculation. Zhou Shaoxian focused

on the navigation performance characteristics of SWATH, and discussed the structural optimization design measures of SWATH from the external load analysis and structural numerical analysis ${ }^{[2]}$.

However, most of the traditional optimization designs only focus on the single performance of ships. which divides the interaction among other performance. Although the single performance has achieved outstanding performance, the overall performance has been poor. Therefore, this paper will use chaos algorithm and genetic algorithm ${ }^{[3]}$, through

Zhanyuan Cheng, Songlin Yang, Chengqi Cai, Wenpeng Cai, Xiaoyu Huang, School of Naval Architecture \& Ocean Engineering, Jiangsu University of Science and Technology, Zhenjiang, Jiangsu, China comparing the fitness function values of the single optimization of rapidity with the fitness function values of the comprehensive optimization of rapidity and maneuverability ${ }^{[4]}$, to obtain the optimal optimization results.

\section{OPTIMIZATION MODEL OF SWATH-USV}

The SWATH USV studied in this paper is an inland watercraft, and its sailing area is relatively small. Considering the factors affecting the performance of SWATH, only its comprehensive performance including rapidity and maneuverability is studied, and a comprehensive optimization mathematical model of navigation performance is established [5]

\section{A. Mathematical Model of Rapid Optimization}

\section{a) Rapidity design variables}

Usually, ship resistance and propulsion efficiency were used to characterize ship rapidity. Therefore, from these two aspects, we selected rapidity design variables. Design variables for resistance: Displacement, Block coefficient, Speed, Ratio of length to diameter of a floating body, Prop length, Hydrofoil parameters; Design variables for propulsion: Propeller diameter, Speed, Pitch ratio. Comprehensive consideration: Length of submersible body, Dive Diameter $\left(D_{1}\right)$, Prop Length $\left(L_{s}\right)$, Maximum Width Of Pillar $\left(t_{s}\right)$, Ship Length ( $\left.L\right)$, Ship Width $(B)$, Draft ( $T$ ), Longitudinal position of center of buoyancy $\left(L_{c p}\right)$, Propeller diameter $\left(D_{P}\right)$, Disc ratio $\left(A_{e o}\right)$, Pitch ratio $\left(P_{D P}\right)$, Propeller speed ( $N$ ), Design speed $\left(V_{S}\right)$, Hydrofoil length ( $Z C$ ), Hydrofoil string length ( $X C$ ), Initial attack angle of hydrofoil $(\alpha)$. Using a vector representation $\left(X_{s p}\right)$,

$$
\begin{aligned}
& \mathrm{X}_{\mathrm{sp}}=\left\{\mathrm{L}_{\mathrm{h}}, \mathrm{D}_{1}, \mathrm{~L}_{\mathrm{s}}, \mathrm{t}_{\mathrm{s}}, \mathrm{L}, \mathrm{B}, \mathrm{T},\right. \\
& \left.\mathrm{L}_{\mathrm{cb}}, \mathrm{D}_{\mathrm{p}}, \mathrm{A}_{\mathrm{eo}}, \mathrm{P}_{\mathrm{dp}}, \mathrm{N}, \mathrm{V}_{\mathrm{s}}, \mathrm{ZC}, \mathrm{XC}, \alpha\right\}
\end{aligned}
$$

\section{b) Rapidity objective function}

The rapidity of ships mainly consists of two aspects: the resistance to hull sailing and the efficiency of ship propulsion. The resistance is highly correlated with the displacement of the ship. In this paper, the optimization of rapidity was selected as the objective function of the resistance under the unit drainage volume:

$$
f_{1}(x)=R_{\text {total }} / \nabla
$$

In this formula, $R_{\text {total }}$ :Total resistance for navigation $(\mathrm{N}) ; \nabla$ :Drainage volume $\left(\mathrm{m}^{3}\right)$

Hull resistance consists of wave making resistance and viscous resistance. 


$$
\begin{aligned}
& R_{\text {total }}=R_{W}+R_{V} \\
& =0.5 \rho V^{2} S\left(C_{W}+C_{V}\right)
\end{aligned}
$$

Among them, the wet surface area of the hull can be estimated by the following formula:

$$
\begin{aligned}
& S=2 \times\left(2.025 L_{s}\left(T-D_{1}\right)\right. \\
& \left.+1.253 \alpha D_{1} L_{h} C_{P}{ }^{0.5}\right)
\end{aligned}
$$

In the formula, $L_{h}{ }^{---}$Floating body length $(m) ; L_{s}---$ Prop length $(m) ; D_{1}---$ Floating body diameter $(m) ; C_{p}---$ Diamond coefficient of main body; $\alpha$--- $\mathrm{A}$ coefficient, $\alpha=3.11+2.095 C_{p}$.

The viscous resistance of SWATH-USV can be divided into three parts.

$$
R_{V 1}=R_{V S}+R_{V B}+R_{A R}
$$

In the formula, $R_{V S}$--- Pillar viscous resistance; $R_{V B}$ Viscous drag of submerged body; $R_{A R}$--- Correlation resistance.

According to this document. The wave resistance coefficient can be expressed by the following formula:

$$
\begin{aligned}
& C_{w}=0.82607-0.90011\left(L_{h} / D_{1}\right)+0.24768 d_{1}-0.048155 L_{s} \\
& +0.15261 t_{s}-0.20488 T_{1}+0.115\left(L_{h} / D_{1}\right)^{2}+0.12008\left(L_{h} / D_{1}\right) \times d_{1} \\
& -0.14762 d_{1}^{2}+0.4391\left(L_{h} / D_{1}\right) \times L_{s}+0.14061 d_{1} \times L_{s}-0.31462 L_{s}^{2} \\
& -0.14308\left(L_{h} / D_{1}\right) \times t_{s}-0.02 d_{1} \times t_{s}+0.067927 L_{s} \times t_{s}-0.06259 t_{s}^{2} \\
& -0.10343\left(L_{h} / D_{1}\right) \cdot T_{1}-0.088095 d_{1} \times T_{1}-0.3455 L_{s} \times T_{1} \\
& +0.027646 t_{s} \times T_{1}+0.28422 T_{1}^{2}
\end{aligned}
$$

In the formula, $L_{h}$---Floating body length $(m) ; D_{1}$---Floating body diameter $(m) ; d_{1}$---The offset of the support relative to the center of the submerged $\operatorname{body}(m) ; L_{s}$--- Prop length $(m) ; t_{s}$--- Maximum width of pillar $(m) ; T_{1}$--- Submerged draught depth (This depth value is the actual water depth minus the maximum radius of the submerged body.)

The propulsion coefficient P.C was selected as a fast-moving part of the objective function:

$$
f_{2}(x)=P . C=\eta_{H} \eta_{R} \eta_{s} \eta_{0}
$$

In the formula: $\eta_{0}{ }^{---}$Propeller open water efficiency; $\eta_{H^{---}}$Hull efficiency; $\quad \eta_{R}{ }^{---}$Relative rotation efficiency; $\eta_{s}{ }^{---}$Shafting efficiency.

To sum up, the total objective function of rapidity is the weighted product of two sub-objective functions, which is expressed in the following formula:

$$
f(x)=f_{1}(x)^{\beta 1} \times f_{2}(x)^{\beta 2}
$$

In the formula, $\beta_{1} \beta_{2}$ mean the Weight number of $f_{1}(x), f_{2}(x)\left(\beta_{1}>0, \beta_{2}>0\right)$.

\section{B. Mathematical model of maneuverability optimization}

a. Manipulative design variables

Ship maneuverability is the capacity of a ship to maintain or change its state of motion. Whether a catamaran can sail straight while sailing is determined by the heading stability. In this paper, the design stability was selected as the research object of maneuverability. Considering comprehensively, the selected design variables are:The length of the boat $(L)$,ship beam( $B$ ),Draft( $T$ ),Block coefficient ( $C_{b}$ ), Water line length $\left(L_{w}\right)$, expressed in XSP, $X_{S P}=\left\{L, B, T, C_{b}, L_{w}\right\}$.

b. Maneuverability objective function

The linear stability was chosen as the objective function of the maneuverability of the catamaran with small water surface. The linear stability was reflected by the linear stability criterion coefficient.

The stability of linear motion $v, r$ is determined by the changing characteristics of state variables.

The stability of the system is directly related to the four acceleration hydrodynamic derivatives $Y_{v}^{\prime}, Y_{r}^{\prime}, N_{v}^{\prime}, N_{r}^{\prime}$ and the four velocity hydrodynamic derivatives. It can be expressed by the ship's linear stability coefficient:

$$
C=Y_{v}^{\prime} N_{r}^{\prime}-N_{v}^{\prime}\left(Y_{r}^{\prime}-m^{\prime}\right)
$$

Where $Y_{v}^{\prime}, Y_{r}^{\prime}, N_{v}^{\prime}, N_{r}^{\prime}$ mean Hydrodynamic derivatives for dimensionless velocity and $m^{\prime}$ means dimensionless hull quality:

$$
\begin{aligned}
& m^{\prime}=m /\left(0.5 \rho L^{3}\right) \\
& =\rho C_{B} L B T /\left(0.5 \rho L^{3}\right) \\
= & 2 C_{B} \frac{B}{L} \frac{T}{L}
\end{aligned}
$$

The hydrodynamic derivatives of the hull can be derived from the regression formula of the linear hydrodynamic derivatives [8]:

$$
\begin{aligned}
& \left(Y_{v}\right)_{\text {hull }}=-0.5 \rho L d V\left(0.5 \pi+1.4 C_{b} B / L\right) \\
& \left(Y_{r}\right)_{\text {hull }}=0.5 \rho L^{2} d V(0.25 \pi) \\
& \left(N_{v}\right)_{\text {hull }}=-0.5 \rho L^{2} d V(0.54 \lambda) \\
& \left(N_{r}\right)_{\text {hull }}=-0.5 \rho L^{3} d V\left(0.54 \lambda-\lambda^{2}\right)
\end{aligned}
$$

Where $\lambda=2 d / L_{w}$ means aspect ratio. The above expressions are dimensionless:

$$
\begin{aligned}
& \left(Y_{v}^{\prime}\right)_{m h}=-d\left(0.5 \pi+1.4 C_{b} B / L\right) \\
& \left(Y_{r}^{\prime}\right)_{m h}=d(0.25 \pi) / L \\
& \left(N_{v}^{\prime}\right)_{m h}=-d(\lambda) / L \\
& \left(N_{r}^{\prime}\right)_{m h}=d\left(0.54 \lambda-\lambda^{2}\right) / L
\end{aligned}
$$

\section{Constraint conditions for ship type performance optimization}

\section{a) Equality constraint}

Equation constraints include static water buoyancy conditions, linear fitting constraints, thrust resistance balance, torque balance. The specific constraints are as follows:

(1) If the hydrostatic floatability is restricted, the ship should satisfy the positive floating state, that is, the discharge volume is equal to the gravity: 


$$
\Delta=\rho L B T C_{b}
$$

(2) The thrust resistance balance constraint is equal to the total thrust of the propeller and the thrust of the hydrofoil:

$$
\begin{aligned}
& N_{p} K_{T} \rho N^{2} D_{P}^{4}(1-t)+F_{x} \\
& =R_{t}+R_{x}
\end{aligned}
$$

3) The torque balance constraint, that is, the torque supplied by the main engine to the propeller is equal to the hydrodynamic torque of the propeller:

$$
\frac{\eta_{R} \eta_{s} P_{s}}{2 \pi N}=K_{Q} \rho N^{2} D_{p}^{5}
$$

\section{b) Inequality constraint}

The propeller needs to meet the cavitation requirement according to Keller equation:

$$
\begin{aligned}
& (1.3+0.3 Z) T_{e} /\left(\left(P_{0}-P_{V}\right) D_{P}^{2}\right) \\
& +K=\left(A_{E} / A_{0}\right)_{\min }
\end{aligned}
$$

The inequality constraints are:

$$
\begin{aligned}
& (1.3+0.3 Z) T_{e} /\left(\left(P_{0}-P_{V}\right) D_{P}^{2}\right) \\
& +K-\left(A_{E} / A_{0}\right) \leq 0
\end{aligned}
$$

In the formula, $P_{0}$ means static pressure at the center of propeller shaft; $P_{V}$ means Vaporization pressure of seawater at 15 degrees Celsius; $T_{e}$ means thrust for propeller; $Z$ means numbers of blades for propeller; $K$ is a constant where Fast ship equals 0 , Other twin oars equal 0.1 and Single paddle ship equals 0.2 .

The range of the 7 design variables.

According to the specifications of sea going ships, the initial stability height, $G M>0.7 m$;

$$
\begin{aligned}
& \text { Rolling cycle: } \\
& T_{\varphi}=1.05 \mathrm{~B} / \sqrt{G M}<6.0 \mathrm{~s}
\end{aligned}
$$

\section{Total objective function}

The objective functions of the three subsystems of UAV, namely, rapidity, maneuverability and seakeeping, were synthesized, and the total objective functions of the synthetical optimization were constructed in the form of power exponential product as follows.

$$
f(x)=f_{1}(x)^{\alpha 1} \bullet f_{2}(x)^{\alpha 2} \bullet f_{3}(x)^{\alpha 3}
$$

Where $\alpha 1, \alpha 2, \alpha 3$ are the weights of the rapidity resistance function, the rapidity propulsion function and the maneuverability function of the unmanned aerial vehicle with the form of the product sum, $\alpha 1 \times \alpha 2 \times \alpha 3=1$. This paper needs comprehensive optimization of performance. The larger the total objective function, the better.

\section{DIFFERENT OPTIMIZATION ALGORITHMS ARE INTRODUCED AND COMPARED}

The optimized object is a piece of SWATH-USV, which refers to the size of the mother ship, and the displacement is set at 6.9T. The captain is about $8 \mathrm{~m}$. The optimization program is carried out on the computer. Various parameters are set according to the prompt. First, the drainage is specified, then the upper and lower limits of the design variables are set according to the figure below, and then the initial weights are set for each optimization subsystem. According to the product of each weight given in the previous chapter is 1 and the importance of each performance, the weight of fast objective function 1, fast objective function 2 is 1 , and the weight of rolling cycle is 0 . Finally, the penalty function is set and the optimization algorithm is selected to calculate.

In this paper, chaos optimization algorithm and genetic algorithm (GA) are used to optimize the calculation.

Chaos refers to the determination of the initial state of the long-term behavior of the dynamic system, or the system parameters that are extremely sensitive, but do not diverge, and they cannot be accurately repeated. It is a complex dynamic behavior of the general non-linear system. Chaotic variables seem to be a chaotic process of change, but in fact they contain intrinsic regularity. By using the randomness, ergodicity and regularity of chaotic variables, the optimization search can be carried out. The basic idea is to map chaotic variables linearly to the value interval of optimization variables, and then search by using chaotic variables. Therefore, chaotic optimization algorithm has the characteristics of ergodicity, universality and inherent randomness.

Genetic algorithm (GA) is a global optimization probabilistic search method with high parallelism, randomness and self-adaptability, which is based on the principles of biogenetics and evolution. The theoretical basis of the effectiveness of genetic algorithm is pattern theorem and building block hypothesis. The pattern theorem guarantees that the sample of the better pattern (the better solution of genetic algorithm) increases exponentially, which satisfies the necessary condition of finding the optimal solution, that is, the possibility of finding the global optimal solution exists in genetic algorithm. The building block hypothesis points out that genetic algorithm has the ability to find the global optimal solution, that is, the mode (building block) with low order, short distance and high average fitness can combine with each other under the action of genetic operators to generate the mode with high order, long distance and high average fitness, and finally generate the global optimal solution.

In this paper, chaotic optimization algorithm and genetic optimization algorithm are used to optimize the weights of the single optimization of rapidity and the comprehensive optimization of rapidity and maneuverability respectively. By comparing the fitness function values obtained, the optimal results of ship form coefficients and the advantages and disadvantages of the single optimization of rapidity and the comprehensive optimization of rapidity and maneuverability are finally obtained.

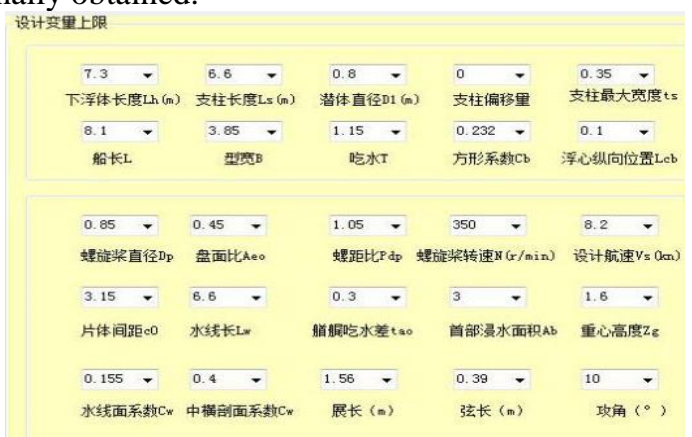

Figure 2.1 Design variable upper bound 


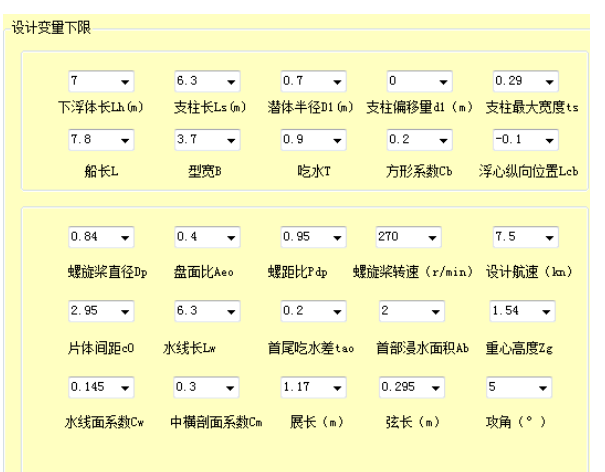

Figure 2.2 Design variable Lower bound

\section{A. The single optimization of rapidity}

a) Chaos optimization algorithm

The basic parameters were set as follows:

Population size: 200, genetic algebra :40000 、 80000 、 $120000 、 160000 \cdot 200000$ ( each generation calculate five times and choose the best result).The optimization results were shown in the following figure.

Table A-1 Optimal calculation results of different optimization algebras with single optimization of rapidity using chaotic algorithm

\begin{tabular}{lccccc}
\hline $\begin{array}{l}\text { Optimizati } \\
\text { on algebra }\end{array}$ & 40000 & 80000 & 120000 & 160000 & 200000 \\
\hline $\begin{array}{l}\text { Fitness } \\
\text { function } \\
\text { value }\end{array}$ & 7.64 & 8.37 & 8.68 & 8.83 & 8.88 \\
$\begin{array}{l}\text { Penalty } \\
\text { function } \\
\text { value }\end{array}$ & 1.00 & 1.00 & 1.00 & 1.00 & 1.00 \\
\hline
\end{tabular}

b) Genetic optimization algorithm

The basic parameters were set as follows:

Population size: 200, Variable carrier probability :0.0001-0.001; Genetic factor 0.5, evolutionary weight 0.5 ; No crossing, no variation, choosing growth mechanism in the calculation strategy ; genetic algebra :1000 、 2000、3000、 4000 -5000( each generation calculate five times and choose the best result ).The optimization results were shown in the following figure.

Table A-2 Optimal calculation results of different optimization algebras with single optimization of rapidity using genetic algorithm

\begin{tabular}{llllll}
\hline $\begin{array}{l}\text { Optimization } \\
\text { algebra }\end{array}$ & 1000 & 2000 & 3000 & 4000 & 5000 \\
\hline $\begin{array}{c}\text { Fitness function } \\
\text { value }\end{array}$ & 8.26 & 8.70 & 9.12 & 9.32 & 9.36 \\
$\begin{array}{c}\text { Penalty function } \\
\text { value }\end{array}$ & 1.00 & 1.00 & 1.00 & 1.00 & 1.00 \\
\hline
\end{tabular}

c) Comparative conclusion

In the case of single optimization rapidity, genetic optimization algorithm has better optimization results than chaotic optimization algorithm, and relatively speaking, the results of genetic optimization algorithm are better.

\section{$B$. The comprehensive optimization of rapidity and maneuverability}

a) Chaos optimization algorithm

The basic parameters were set as follows:

Population size: 200, genetic algebra :40000 、 80000 、 $120000 、 160000 \cdot 200000$ ( each generation calculate five times and choose the best result).The optimization results were shown in the following figure.

Table B-3 Optimal calculation results of different optimization algebras with comprehensive optimization of rapidity and maneuverability using chaotic algorithm

\begin{tabular}{llllll}
\hline Optimization algebra & 40000 & 80000 & 120000 & 160000 & 200000 \\
\hline Fitness function value 9.3 & 9.64 & 10.81 & 11.05 & 11.06 \\
Penalty function value 1.00 & 1.00 & 1.00 & 1.00 & 1.00 \\
\hline
\end{tabular}

b) Genetic optimization algorithm

The basic parameters were set as follows:

Population size: 200, Variable carrier probability :0.0001-0.001; Genetic factor 0.5, evolutionary weight 0.5 ; No crossing, no variation, choosing growth mechanism in the calculation strategy ; genetic algebra :1000 、 2000 、3000 、 4000 -5000( each generation calculate five times and choose the best result ).The optimization results were shown in the following figure.

Table B-4 Optimal calculation results of different optimization algebras with comprehensive optimization of rapidity and maneuverability using genetic algorithm

\begin{tabular}{llllll}
\hline Optimization algebra & 1000 & 2000 & 3000 & 4000 & 5000 \\
\hline Fitness function value 10.06 & 10.49 & 11.25 & 11.65 & 11.72 \\
Penalty function value 1.00 & 1.00 & 1.00 & 1.00 & 1.00 \\
\hline
\end{tabular}

\section{Contrast result}

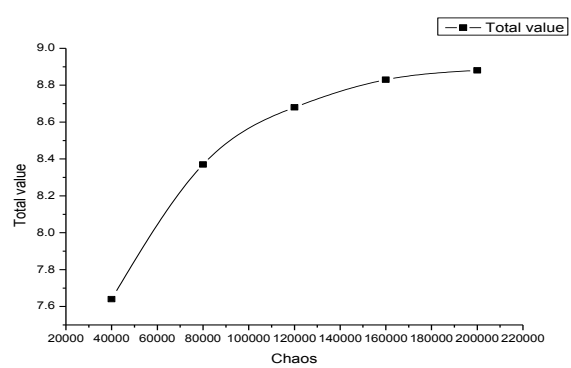

Figure 2-3 Single optimization of rapidity using chaotic algorithm

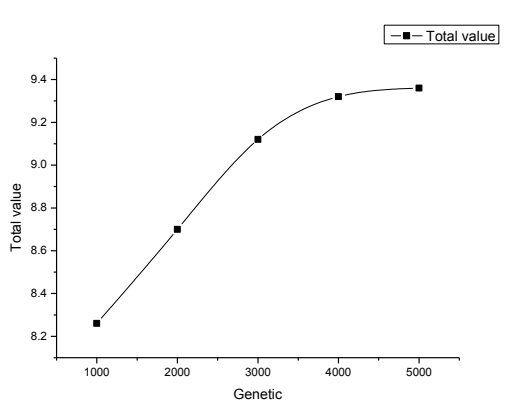

Figure 2-4 Single optimization of rapidity using genetic algorithm 
International Journal of Engineering and Applied Sciences (IJEAS)

ISSN: 2394-3661, Volume-6, Issue-7, July 2019

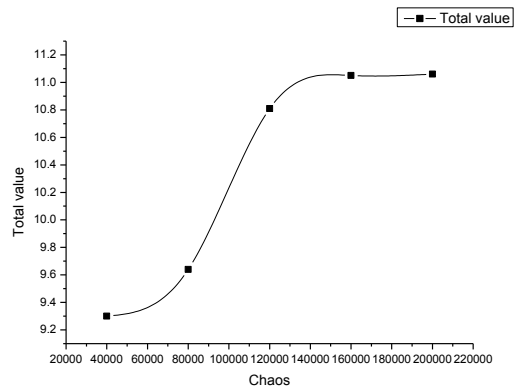

Figure 2-5 Comprehensive optimization of rapidity and maneuverability using chaotic algorithm

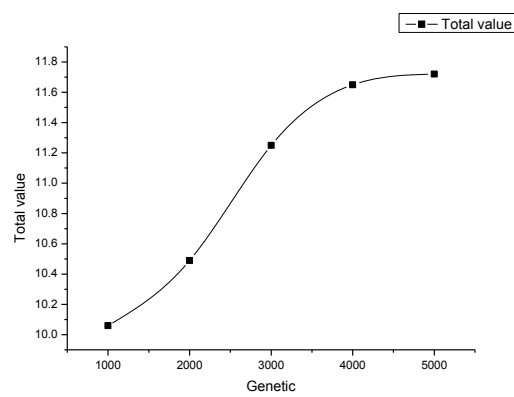

Figure2-6 Comprehensive optimization of rapidity and maneuverability using genetic algorithm

In Table 2-1, Table 2-2, Table 2-3 and Table 2-4, the penalty function values are all 1 , indicating that all constraints are satisfied. In addition, from the results of the fitness function optimization in Figure2-3,Figure2-4,Figure2-5, Figure2-6,it can be concluded that:

(1) The genetic optimization algorithm is superior to the chaotic optimization algorithm, and the stability of the optimization results is higher than that of the chaotic optimization algorithm.

(2) The fitness function value obtained by the combination of rapidity and maneuverability weights is higher than that obtained by the single optimization rapidity, and the genetic algebra is 5000 generations.

1)D. Optimization results

The optimum ship form parameters and the optimum function values are shown in the tables below.

Table C-6 Optimal results of ship form parameters

\begin{tabular}{|c|c|c|c|c|}
\hline number & design variabe & $\begin{array}{l}\text { lower } \\
\text { limit }\end{array}$ & $\begin{array}{l}\text { Upper } \\
\text { limit }\end{array}$ & $\begin{array}{c}\text { Optimal } \\
\text { value }\end{array}$ \\
\hline 1 & Submergence length $L_{h}(\mathrm{~m})$ & 7 & 7.3 & 7.043 \\
\hline 2 & Prop length $L_{s}(\mathrm{~m})$ & 6.3 & 6.6 & 6.359 \\
\hline 3 & Submersible radius $D 1(\mathrm{~m})$ & 0.7 & 0.8 & 0.764 \\
\hline 4 & Maximum width of pillar $t s$ & 0.29 & 0.35 & 0.342 \\
\hline 5 & ship' $^{\prime}$ s longitude $L(\mathrm{~m})$ & 7.8 & 8.1 & 8.07 \\
\hline 6 & $\begin{array}{c}\text { ship beam } \\
B(\mathrm{~m})\end{array}$ & 3.7 & 3.85 & 3.828 \\
\hline 7 & $\begin{array}{l}\text { Draft } \\
T(\mathrm{~m})\end{array}$ & 0.9 & 1.15 & 0.96 \\
\hline 8 & $\begin{array}{l}\text { block coefficient } \\
\qquad \mathrm{Cb}\end{array}$ & 0.2 & 0.232 & 0.227 \\
\hline 9 & $\begin{array}{c}\text { lengthwise position } \\
\text { of buoyancy } \\
L c p(\mathrm{~m})\end{array}$ & -0.1 & 0.1 & 0.042 \\
\hline 10 & $\begin{array}{l}\text { Propeller diameter } \\
\qquad D p(\mathrm{~m})\end{array}$ & 0.835 & 0.85 & 0.848 \\
\hline
\end{tabular}

11

14

15

16

17

18

19

20

21

22

Table C-7 Optimization function value of optimal results

System total objective function and sub objective function

\begin{tabular}{cc}
\hline Fitness function value & 11.7206507103145 \\
Total objective & 11.7206507103145 \\
function value & \\
Degree of buoyancy & $99.93 \%$ \\
constraint satisfaction & Degree of constraint \\
satisfaction & $99.91 \%$ \\
Degree of torque & $99.12 \%$ \\
constraint satisfaction & Penalty function value
\end{tabular}

\section{CONCLUSION}

In this paper, according to the parameters that affect the comprehensive performance of SWATH-USV, a comprehensive optimization mathematical model of SWATH-USV is established, and then the objective function of each performance is constructed as a comprehensive optimization total objective function in the form of power exponential product, and the constraints are constructed by combining design variables and actual conditions.By discussing the selection of chaotic optimization algorithm and genetic optimization algorithm, as well as the weights of rapidity and maneuverability, the optimal ship form parameters for the overall performance of SWATH are finally obtained. The following conclusions can be drawn:

(1) By comparing the fitness function values of genetic optimization algorithm and chaotic optimization algorithm, we can see that the optimization results of genetic optimization algorithm are better and more stable.

(2) Using genetic algorithm with single growth mechanism, the fitness function increases with the increase of the number of inheritances, and finally tends to be stable after 4000 generations. When the number of inheritances is about 5000 generations, the overall optimization effect is the best. 
(3) When optimizing ship form parameters, considering the weights of rapidity and maneuverability, a comprehensive optimization can achieve better results.

\section{REFERENCES}

[1] Wei Keke, Gao Xiaopeng, Dong Zushun.Study on Resistance Performance of New Type Small Waterline Surface Wave-piercing Catamaran[J]. Shipbuilding of China, 2018, 59(03):126-136.

[2] Zhou Shaoxian.Study on Structure and Optimum Design of Small Waterline Surface Catamaran[J]. China Water Transport, 2017(11):34-35.

[3] Wei Zifan, Jing Shengping, Yang Songlin.Performance optimization analysis of new surface unmanned aerial vehicle based on improved genetic algorithm[J].Journal of Jiangsu University of Science and Technology (Natural Science Edition), 2017, 31 (01): 6-11.

[4] Wang Mengyun, Yu Qiang, Yang Songlin. Comprehensive optimization analysis of UUV rapidity and energy system based on genetic algorithm[J]. Ship Science and Technology,2015,37(02):73-78.

[5] Cao Xue. Preliminary Comprehensive Optimization Analysis of SWATH-USV Ship Type with All Green Energy[D]. Journal of Jiangsu University of Science and Technology (Natural Science Edition), 2017.

[6] Campana E F, Diez M , Liuzzi G, et al. A multi-objective DIRECT, algorithm for ship hull optimization[J]. Computational Optimization and Applications, 2018, 71(1):53-72.

[7] Lee C M, CURPHEY R M. Prediction of motion, stability, and wave load of small-waterplane-area twin-hull ships [J]. Trans,SNAME, 1977,(85):94- 130.

[8] Deng Fang, Deng Weibin. Calculation of Resistance Performance and Design Optimization of Catamaran [J]. Journal of Qingdao University of Science and Technology(Natural Science Edition), 2015, 36(01): 72-6. 